\title{
Energy efficiency analysis of relay-assisted cellular networks
}

\author{
Huan $\mathrm{Yu}^{1,2}$, Yunzhou Li ${ }^{2 *}$, Marios Kountouris ${ }^{3},{\text { Xibin } X \mathrm{U}^{2} \text { and Jing Wang }}^{2}$
}

\begin{abstract}
To meet the demand for higher throughput, improved coverage and enhanced reliability, future wireless cellular networks face significant technical challenges. One promising solution is to place relay stations between transmitters and receivers in the cellular network. Meanwhile, as energy consumption reduction has been an important concern for the wireless industry, energy-efficient communications is of prime interest for future networks. In this paper, we study whether and how relays can improve the energy efficiency of cellular networks. Specifically, the energy efficiency of relay-assisted cellular networks is analyzed using tools of stochastic geometry. We first derive the coverage probability for the macro base station (MBS) to user (UE), the MBS to relay station (RS), and the RS to UE links, and then we model the power consumption at the MBS and RS. Based on the analytical model and expressions, the energy efficiency of relay-assisted cellular networks is then evaluated and is shown to be strictly quasi-concave on the transmit power for MBS to UE link or the RS to UE link. Numerical results show that the energy efficiency first improves while it hits a ceiling as the MBS density increases.
\end{abstract}

\section{Introduction}

With the exponential growth of wireless traffic, future cellular networks face huge challenges for catering higher data rate and transmission reliability. One effective and promising way to meet the traffic demands is to deploy relay stations (RSs) in the traditional cellular networks, as a means to increase coverage, throughput, and reliability [1]. Meanwhile, as the energy consumption reduction has become a global trend, the research for energy-efficient wireless networks has increasingly attracted attention recently [2].

There have been some works in the literature about energy-efficient relay-assisted networks from the link layer to the network layer [3-8]. For the link layer, the energy efficiency problem for multi-carrier amplify-andforward (AF) relay links is considered in [3]. The optimization problem cast in the paper is non-convex and is converted into a quasi-concave problem by two approximate methods, thus providing a suboptimal solution. In

\footnotetext{
${ }^{*}$ Correspondence: liyunzhou@mail.tsinghua.edu.cn

2 Research Institute of Information Technology, State Key Laboratory on Microwave and Digital Communications, Tsinghua National Laboratory for Information Science and Technology(TNList), Tsinghua University, 100084 Beijing, China

Full list of author information is available at the end of the article
}

[4-6], energy-efficient two-way relay links under different forwarding schemes are studied. The energy efficiency for one-way relay transmission and two-way relay transmission is compared when the AF strategy is adopted in [4], showing that when the bidirectional data amounts are equal, two-way relay transmission performs better; otherwise one-way relay transmission may offer higher energy efficiency. In [5], hybrid relay transmission transmitting partial messages with one-way relay transmission and the remaining messages with two-way relay transmission is proposed. Simulation results show that the performance of hybrid relay transmission is better than that of the two-way relay transmission and one-way relay transmission. Energy efficiency of the two-way relay link using decode-and-forward (DF) strategy is investigated in [6] and an improved bisection algorithm is proposed to find the optimal energy efficiency. For the network layer, in $[7,8]$, energy-efficient multi-relay networks are studied, in which the source node sends massage to the destination node via the relay nodes and the best one or several relay nodes by a certain criterion are selected to forward the message from source node in order to achieve the highest energy efficiency.

In previous studies on the performance of traditional cellular networks, the cell shape is often supposed to be hexagonal or square, which may not be the case in

\section{Springer}

(c) 2014 Yu et al.; licensee Springer. This is an Open Access article distributed under the terms of the Creative Commons

Attribution License (http://creativecommons.org/licenses/by/2.0), which permits unrestricted use, distribution, and reproduction in any medium, provided the original work is properly cited. 
realistic scenarios (e.g., due to shadowing) or in heterogeneous and uncoordinated networks. Besides, complex time-consuming system-level simulations must be used. In [9], an approach to derive the coverage and rate in cellular networks based on stochastic geometry is proposed, which is more tractable compared to the traditional grid models. Under this approach, in [10], the design of energy-efficient heterogeneous cellular networks through the employment of base station sleeping mode strategies as well as small cells are investigated, in which some methods can be considered in the relay-assisted cellular networks. The relationship among the energy efficiency and the intensity of non-cooperative users (UEs) and cooperative UEs is studied in [11] based on the stochastic geometry approach. In [12], the effect of base station density on the energy efficiency of relay-assisted cellular networks is investigated using the stochastic geometry model. However, the paper only considers the special case where the path loss exponent $\alpha=4$; while in our work, we derive expressions that are valid for any $\alpha>2$.

In our work, we analyze the energy efficiency for relayassisted cellular networks using models based on stochastic geometry [9], where the macro base stations (MBSs) and relay stations(RSs) locations are distributed according to independent homogeneous Poisson point process (HPPP). The mobile users (UEs) in the network are divided into two types, i.e., UEs that communicate directly with MBSs (M-UEs) and UEs that communicate with MBSs via the help of RSs (R-UEs). The main contributions of this paper are summarized as follows:

- Building on [9], a downlink relay-assisted cellular network model is proposed based on the stochastic geometry theory.

- The coverage probabilities for the MBS-RS, the MBSUE, and the RS-UE links are derived, and the power consumption for MBSs as well as RSs are modeled.

- The energy efficiency expression for relay-assisted cellular networks is analytically deduced under specific power consumption models.

- The energy efficiency is proved to be strictly quasiconcave on the transmit power for MBS to UE link or RS to UE link, and an algorithm based on bisection method is proposed to find the optimal value.

The rest of this paper is organized as follows: in Section 2, the system model is described. In Section 3, the energy efficiency for the downlink relay-assisted cellular networks is derived. Simulation results are shown in Section 4, and conclusions are given in Section 5.

\section{System model}

We consider a relay-assisted cellular network where the MBSs and RSs are located according to independent
HPPPs $\Theta_{M}$ and $\Theta_{R}$ with densities $\lambda_{M}$ and $\lambda_{R}$, respectively, in the Euclidean plane. Half-duplex relay nodes using DF strategy are considered, where each RS connects to the geographically closest MBS and has coverage of a disk of radius $R$, i.e., the RSs deployed in the Voronoi cell of an MBS are connected with it (cf. Figure 1). UEs that communicate directly with the MBS (M-UE) are distributed according to some independent stationary point process and connect with the closest MBS. Meanwhile, UEs that communicate with the MBS via the RS (R-UE) are arranged with reference to some independent stationary point process within each RS's circular area. Note that these two different types of UEs are not distinguished in Figure 1.

The distance between M-UE (or RS) and the target MBS is denoted by $r$. As $\Theta_{M}$ is a HPPP with density $\lambda_{M}$, the probability density function (pdf) of $r$ is $f_{r}(r)=$ $2 \pi \lambda_{M} r \exp \left(-\pi \lambda_{M} r^{2}\right)$ [9]. Furthermore, since the distribution of RSs follows a HPPP and R-UEs are distributed according to some independent stationary point process within each relay's circular area, the distance between the R-UE and its corresponding RS $r$ follows a distribution with $\operatorname{pdf} f_{R}(r)=2 r / R^{2}$.

As shown in Figure 2, in each cell, the MBS-UE link and the MBS-RS-UE link work in non-overlapping frequency bands with bandwidth $\omega_{M}$ and $\omega_{R}$, respectively. For the MBS-RS-UE link with half-duplex relays, the transmission is divided into two phrases. In the first phrase, the MBS sends messages to the RS, and in the second phrase, the RS decodes the messages and forwards them to the user. The time duration of both phases is equal. We assume that each MBS serves at most one M-UE and RS at any time. If more than one M-UE (RS) are located in the MBS's cell, orthogonal resource sharing such as time division multiple access (TDMA) is performed. Similarly, each RS serves one R-UE at any time and TDMA may be used if multiple R-UEs are distributed in the RS's serving area. Once TDMA is selected, the MBS can schedule the resource and select the RS to work with, the RSs only need to know which one is selected.

\section{Energy efficiency analysis}

In this section, the coverage probabilities are first derived, and then, the energy efficiency expression is given for certain power consumption models.

\subsection{Coverage probability}

The signal-to-interference-plus-noise ratio (SINR) in the downlink from the transmitter (MBS or RS) $s$ to the receiver (RS or UE) $d$ is given by

$$
\operatorname{SINR}(s \rightarrow d)=\frac{P h r^{-\alpha}}{I+\sigma^{2}}
$$




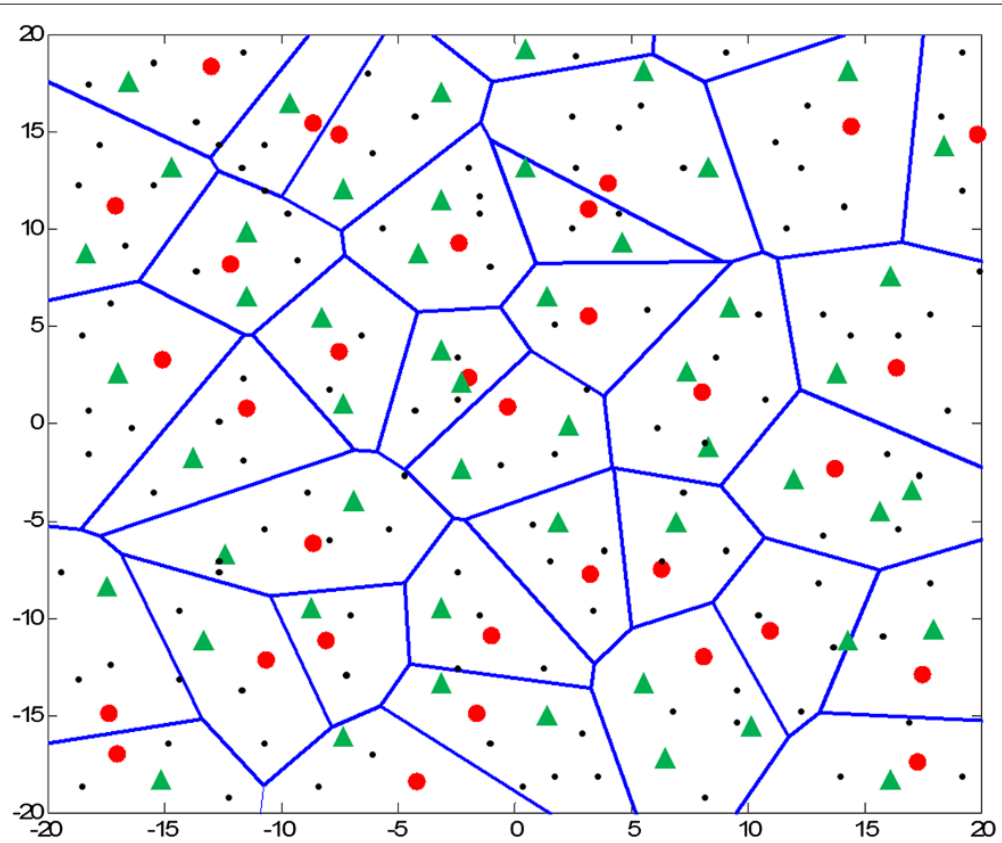

Figure 1 HPPP distributed MBSs and RSs with two types of UEs. Big dots, MBSs; triangles, RSs; little dots, UEs.

where $P$ is the transmit power for node $s, h$ is the channel power gain due to the small-scale fading, $r$ is the distance between $s$ and $d, \alpha$ is the path loss exponent, $I$ is the aggregate interference from all the other active transmitters operating in the same frequency band, and $\sigma^{2}$ is the variance of the additive white Gaussian background noise.
All channels are assumed to be subject to Rayleigh fading, i.e., $h \sim \exp (1)$.

The coverage probability is defined as the probability that the receive SINR is above a certain threshold $\Gamma$ and can be written as

$$
p_{c}=\mathbb{P}(\operatorname{SINR} \geq \Gamma)
$$

\section{Frequency}

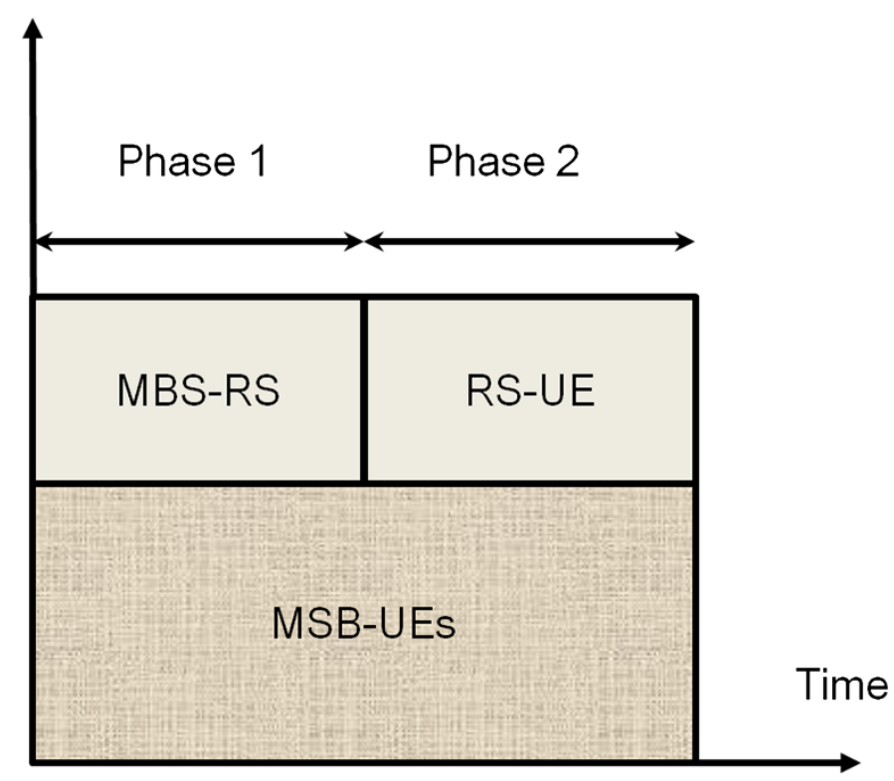

Figure 2 Relay-assisted cellular network frame structure. 


\subsubsection{Coverage probability for the MBS-UE link}

First, we assume that M-UEs that are directly connected to MBS are distributed according to an independent stationary point process. Second, the transmit power from MBS to $\mathrm{M}-\mathrm{UE}$ is $P_{\mathrm{MU}}$, the receive threshold is denoted as $\Gamma_{\mathrm{MU}}$, and $I_{\mathrm{MU}}$ is the aggregate interference from all the other active MBSs except the target $\mathrm{MBS} m_{0}$. Then, the coverage probability for a typical MBS-UE link is defined as

$$
p_{c_{-} \mathrm{MU}}=\mathbb{P}\left(\mathrm{SINR}_{\mathrm{MU}} \geq \Gamma_{\mathrm{MU}}\right)
$$

where SINR $\mathrm{MU}=\frac{P_{\mathrm{MU}} h_{0} r^{-\alpha}}{I_{\mathrm{MU}}+\sigma^{2}}$ and $I_{\mathrm{MU}}=\sum_{i \in \Theta_{M} \backslash\left\{m_{0}\right\}} P_{\mathrm{MU}} h_{i} r_{i}^{-\alpha}$ and $f_{r}(r)=2 \pi \lambda_{M} r \exp \left(-\pi \lambda_{M} r^{2}\right)$.

Theorem 1. The coverage probability for the MBS-UE link $p_{c_{-} \mathrm{MU}}$ is given by

$$
\begin{aligned}
p_{c_{-} \mathrm{MU}}= & 2 \pi \lambda_{M} \int_{r>0} \exp \left(-\pi \lambda_{M} r^{2}\left(1+\rho\left(\Gamma_{\mathrm{MU}}, \alpha\right)\right)\right) \\
& \times \exp \left(-\Gamma_{\mathrm{MU}} r^{\alpha} \sigma^{2} / P_{\mathrm{MU}}\right) r \mathrm{~d} r
\end{aligned}
$$

where $\rho\left(\Gamma_{\mathrm{MU}}, \alpha\right)=\Gamma_{\mathrm{MU}}^{2 / \alpha} \int_{\Gamma_{\mathrm{MU}}^{-2 / \alpha}}^{\infty} \frac{1}{1+v^{\alpha / 2}} \mathrm{~d} v$.

In the interference-limited regime where the background thermal noise is ignored $\left(\sigma^{2} \rightarrow 0\right)$, the coverage probability can be simplified as $p_{c_{-} \mathrm{MU}}=\frac{1}{1+\rho\left(\Gamma_{\mathrm{MU}}, \alpha\right)}$.

Proof. The details of the derivation can be found in [9], and we just provide here the key points. From Equation 3, the definition of SINR and the assumption that $h_{0} \sim$ $\exp (1)$, the coverage probability can be rewritten as

$$
\begin{aligned}
p_{c_{-} \mathrm{MU}}= & \mathbb{P}\left(h_{0} \geq \Gamma_{\mathrm{MU}} r^{\alpha}\left(I_{\mathrm{MU}}+\sigma^{2}\right) / P_{\mathrm{MU}}\right) \\
= & \int_{r>0} \mathbb{P}\left(h_{0} \geq \Gamma_{\mathrm{MU}} r^{\alpha}\left(I_{\mathrm{MU}}+\sigma^{2}\right) / P_{\mathrm{MU}} \mid r\right) f_{r}(r) \mathrm{d} r \\
= & \int_{r>0} \exp \left(-\Gamma_{\mathrm{MU}} r^{-\alpha} \sigma^{2} / P_{\mathrm{MU}}\right) L_{I_{\mathrm{MU}}} \\
& \times\left(\Gamma_{\mathrm{MU}} r^{\alpha} / P_{\mathrm{MU}}\right) f_{r}(r) \mathrm{d} r
\end{aligned}
$$

where $L_{I_{\mathrm{MU}}}(s)$ is the Laplace transform of $I_{\mathrm{MU}}$.

Considering the definition of Laplace transform and the assumption that $h_{i} \sim \exp (1), L_{I_{\mathrm{MU}}}(s)$ is given by

$$
\begin{aligned}
L_{I_{\mathrm{MU}}}(s) & =\mathbb{E}_{I_{\mathrm{MU}}}\left[e^{-s I_{\mathrm{MU}}}\right] \\
& =\exp \left(-2 \pi \lambda_{M} \int_{r}^{\infty}\left(1-\frac{1}{1+s P_{\mathrm{MU}} u^{-\alpha}}\right) u \mathrm{~d} u\right)
\end{aligned}
$$

The result of Equation 5 is deduced from the property of the probability generating functional (PGFL) for PPP, which for some function $g(x)$, it satisfies $\mathbb{E}\left[\prod_{x \in \Theta} g(x)\right]=$ $\exp \left(-\lambda \int_{R^{2}}(1-g(x)) \mathrm{d} x\right)$ [13], and $\lambda$ is the density of the interference nodes.
Combining the results of Equations 5 and 6, Equation 4 is obtained.

When $\sigma^{2} \rightarrow 0$, it can be easily derived for Equation 4 that $p_{c \_\mathrm{MU}}=\frac{1}{1+\rho\left(\Gamma_{\mathrm{MU}, \alpha}\right)}$.

Using the Theorem 1, the following corollaries can be easily obtained.

Corollary 1. In the interference-limited regime, the coverage probability $p_{c_{-} \mathrm{MU}}$ increases with increasing $P_{\mathrm{MU}}$, and when $P_{\mathrm{MU}}$ goes to infinity, $\lim _{P_{\mathrm{MU} \rightarrow \infty}} p_{c_{-} \mathrm{MU}}=\frac{1}{1+\rho\left(\Gamma_{\mathrm{MU}}, \alpha\right)}$.

Corollary 2. With $\sigma^{2}>0$, the coverage probability $p_{c_{-} \mathrm{MU}}$ decreases with increasing $\Gamma_{\mathrm{MU}}$.

\subsubsection{Coverage probability for the MBS-RS link}

Suppose the relay can successfully decode the signals from the MBS if the received SINR is larger than or equal to $\Gamma_{\mathrm{MR}}$, then the coverage probability for the MBS-RS link can be written as

$$
p_{c \_\mathrm{MR}}=\mathbb{P}\left(\mathrm{SINR}_{\mathrm{MR}} \geq \Gamma_{\mathrm{MR}}\right)
$$

where $\mathrm{SINR}_{\mathrm{MR}}=\frac{P_{\mathrm{MR}} g_{0} r^{-\alpha}}{I_{\mathrm{MR}}+\sigma^{2}}, I_{\mathrm{MR}}=\sum_{i} P_{\mathrm{MR}} g_{i} r_{i}^{-\alpha}$, for $i$ in all other active MRSs in the MBS-RS frequency band except the target MBS $m_{0}$.

Theorem 2. The coverage probability for the MBS-RS link $p_{c \_ \text {MR }}$ is given by

$$
\begin{aligned}
p_{c \_M R}= & 2 \pi \lambda_{M} \int_{r>0} \exp \left(-\pi r^{2}\left(\lambda_{M}+\lambda \rho\left(\Gamma_{\mathrm{MR}}, \alpha\right)\right)\right) \\
& \times \exp \left(-\Gamma_{\mathrm{MR}} r^{\alpha} \sigma^{2} / P_{\mathrm{MR}}\right) r \mathrm{~d} r
\end{aligned}
$$

where $\lambda=\min \left\{\lambda_{M}, \lambda_{R}\right\}$ and $\rho\left(T_{\mathrm{MR}}, \alpha\right)=\Gamma_{\mathrm{MR}}^{2 / \alpha} \int_{\Gamma_{\mathrm{MR}}^{-2 / \alpha}}^{\infty}$ $\frac{1}{1+v^{\alpha / 2}} \mathrm{~d} v$.

In the interference-limited regime, i.e., $\sigma^{2} \rightarrow 0$, the coverage probability can be simplified as $p_{c_{-} \mathrm{MR}}=$ $\lambda_{M}$

The derivation of the coverage probability $p_{c \_\mathrm{MR}}$ is almost the same as that of $p_{c_{-} M U}$ in the previous subsection except that the density used for the derivation of the Laplace transform of $I_{M R}$ is $\lambda$ instead of $\lambda_{M}$. As we assume that each MBS serves at most one RS at any time in each cell, the density of interference nodes is the smaller of $\lambda_{M}$ and $\lambda_{R}$. Thus, the density used for the derivation of the Laplace transform of $I_{\mathrm{MR}}$ is $\lambda=\mathrm{min}$ $\left\{\lambda_{M}, \lambda_{R}\right\}$.

Similar corollaries can be obtained as follows 
Corollary 3. With $\sigma^{2}>0$, the coverage probability $p_{c_{-} \mathrm{MR}}$ increases with increasing $P_{\mathrm{MR}}$, and when $P_{\mathrm{MR}}$ goes to infinity, $\lim _{P_{\mathrm{MR}} \rightarrow \infty} p_{c_{-} \mathrm{MR}}=\frac{\lambda_{M}}{\lambda_{M}+\lambda \rho\left(\Gamma_{\mathrm{MR}}, \alpha\right)}$.

Corollary 4. With $\sigma^{2}>0$, the coverage probability $p_{c \_\mathrm{MR}}$ decreases with increasing $\Gamma_{\mathrm{MR}}$.

\subsubsection{Coverage probability for the RS-UE link}

We assume that R-UEs receiving messages by the aid of RSs are located according to some stationary point process in a circular area around the RS and of radius $R$. We denote $P_{\mathrm{R}}$ as the transmit power of the RS, $\Gamma_{\mathrm{RU}}$ as the receive threshold, and $I_{\mathrm{RU}}$ as the sum of interference of all other active RSs except the target RS $r_{0}$. The coverage probability for a typical RS-UE link is defined as

$$
p_{c_{-} \mathrm{RU}}=\mathbb{P}\left(\mathrm{SINR} \mathrm{RU} \geq \Gamma_{\mathrm{RU}}\right)
$$

where $\operatorname{SINR}_{\mathrm{RU}}=\frac{P_{\mathrm{R}} l_{0} r^{-\alpha}}{I_{\mathrm{RU}}+\sigma^{2}}$ and $I_{\mathrm{RU}}=\sum_{i} P_{\mathrm{R}} l_{i} r_{i}^{-\alpha}$, for $i$ denoting all other active RSs except $r_{0}$.

As the distance between RS and UE follows a different distribution, the form of $p_{c_{-} \mathrm{RU}}$ is not the same as for $p_{c_{-} \mathrm{MR}}$ and $p_{c_{-} \mathrm{MU}}$.

Theorem 3. The coverage probability for the MBS-RS link $p_{c_{-} \mathrm{RU}}$ is given by

$$
\begin{aligned}
p_{c \_\mathrm{RU}}= & \frac{2}{R^{2}} \int_{0}^{R} \exp \left(-\pi \lambda r^{2} p_{c_{-} \mathrm{MR}} \mu\left(\Gamma_{\mathrm{RU}}, \alpha\right)\right) \\
& \times \exp \left(-\frac{\Gamma_{\mathrm{RU}} r^{\alpha} \sigma^{2}}{\left(1+\Gamma_{\mathrm{RU}}\right) P_{\mathrm{R}}}\right) r \mathrm{~d} r
\end{aligned}
$$

where $\lambda=\min \left\{\lambda_{M}, \lambda_{R}\right\}$ and $\mu\left(\Gamma_{\mathrm{RU}}, \alpha\right)=\left(\frac{\Gamma_{\mathrm{RU}}}{1+\Gamma_{\mathrm{RU}}}\right)^{2 / \alpha}$ $\int_{0}^{\infty} \frac{1}{1+v^{\alpha / 2}} \mathrm{~d} v$.

In the interference-limited regime, the coverage probability can be expressed $a_{s p} p_{-} \mathrm{RU}=\frac{1+\rho\left(\Gamma_{\mathrm{MR}}, \alpha\right)}{\lambda \pi R^{2} \mu\left(\Gamma_{\mathrm{RU}}, \alpha\right)}(1-\exp$ $\left.\left(-\frac{\lambda \pi R^{2} \mu\left(\Gamma_{\mathrm{RU}}, \alpha\right)}{1+\rho\left(\Gamma_{\mathrm{MR}}, \alpha\right)}\right)\right)$.

Proof. From the definition of $p_{c_{-} \mathrm{RU}}$, $\mathrm{SINR}_{\mathrm{RU}}$, and $I_{\mathrm{RU}}$, we can rewrite $p_{c_{-} \mathrm{RU}}$ as

$$
\begin{aligned}
p_{c \_\mathrm{RU}} & =\mathbb{P}\left(\frac{P_{\mathrm{R}} l_{0} r^{-\alpha}}{I_{\mathrm{RU}}+\sigma^{2}} \geq \Gamma_{\mathrm{RU}}\right) \\
& =\int_{0}^{R} \mathbb{P}\left(l_{0} \geq \Gamma_{\mathrm{RU}} r^{\alpha}\left(I_{\mathrm{RU}}+\sigma^{2}\right) / P_{\mathrm{R}} \mid r\right) f_{R}(r) \mathrm{d} r .
\end{aligned}
$$

For analytical convenience, we assume that $\tilde{I}_{\mathrm{RU}}=$ $I_{\mathrm{RU}}+P_{\mathrm{R}} l_{0} r^{-\alpha}$, which encompasses the signals from all the transmitting RSs including $r_{0}$. We substitute $I_{\mathrm{RU}}$ with
$\left(\tilde{I}_{\mathrm{RU}}-P_{\mathrm{R}} l_{0} r^{-\alpha}\right)$ and the coverage probability $p_{c \_\mathrm{RU}}$ is given by

$$
\begin{aligned}
p_{c \_\mathrm{RU}}= & \int_{0}^{R} \mathbb{P}\left(l_{0} \geq \frac{\Gamma_{\mathrm{RU}} r^{\alpha}\left(\tilde{I}_{\mathrm{RU}}+\sigma^{2}\right)}{\left(1+\Gamma_{\mathrm{RU}}\right) P_{R}} \mid r\right) f_{R}(r) \mathrm{d} r \\
\stackrel{(a)}{=} & \frac{2}{R^{2}} \int_{0}^{R} \mathbb{E}_{\tilde{I}_{\mathrm{RU}}}\left[\exp \left(-\frac{\Gamma_{\mathrm{RU}} r^{\alpha}\left(\tilde{I}_{\mathrm{RU}}+\sigma^{2}\right)}{\left(1+\Gamma_{\mathrm{RU}}\right) P_{R}}\right)\right] r \mathrm{~d} r \\
= & \frac{2}{R^{2}} \int_{0}^{R} \exp \left(-\frac{\Gamma_{\mathrm{RU}} r^{\alpha} \sigma^{2}}{\left(1+\Gamma_{\mathrm{RU}}\right) P_{R}}\right) L_{\tilde{I}_{\mathrm{MR}}} \\
& \times\left(\frac{\Gamma_{\mathrm{RU}} r^{\alpha}}{\left(1+\Gamma_{\mathrm{RU}}\right) P_{R}}\right) r \mathrm{~d} r
\end{aligned}
$$

where step ( $a$ ) follows from the fact that $f_{R}(r)=2 r / R^{2}$ and $l_{0} \sim \exp (1)$.

The derivation of $L_{\tilde{I}_{\mathrm{MR}}}$ is similar to that of $L_{I_{\mathrm{MU}}}$ except some differences as shown below.

$$
\left.L_{I_{\mathrm{RU}}}\left(\Gamma_{\mathrm{RU}} r^{\alpha} / P_{R}\right)=\exp \left(-\pi r^{2} \lambda p_{c_{-} \mathrm{MR}}\right) \mu\left(\Gamma_{\mathrm{RU}}, \alpha\right)\right)
$$

where $\lambda=\min \left\{\lambda_{M}, \lambda_{R}\right\}$ and $\mu\left(\Gamma_{\mathrm{RU}}, \alpha\right)=\Gamma_{\mathrm{RU}}^{2 / \alpha} \int_{0}^{\infty}$ $\frac{1}{1+\nu^{\alpha / 2}} \mathrm{~d} \nu$.

One difference is the density used in the expression. At any time, there is at most only one RS communicating with the target MBS, and only the RSs with received SINR larger than the target SINR $\Gamma_{M R}$ forward messages to the R-UEs, so the density used in the above formula is $\lambda p_{c_{-}} \mathrm{MR}$.

Besides, the distance between R-UE and active RSs can be any value, so the integration limits start form 0 (not $r$ ) to $\infty$ in $\mu\left(\Gamma_{\mathrm{RU}}, \alpha\right)$.

Combining Equations 11 and 13, the expression of $p_{c_{-} \mathrm{RU}}$ is obtained.

When $\sigma^{2} \rightarrow 0, p_{c_{-} \mathrm{MR}}=\frac{1}{1+\rho\left(\Gamma_{\mathrm{MR}, \alpha}\right)}$ and then $p_{c_{\_} \mathrm{RU}}$ can be written as

$$
\begin{aligned}
p_{c_{-} \mathrm{RU}} & =\frac{2}{R^{2}} \int_{0}^{R} \exp \left(-\pi r^{2} \lambda \frac{\mu\left(\Gamma_{\mathrm{RU}}, \alpha\right)}{1+\rho\left(\Gamma_{\mathrm{MR}}, \alpha\right)}\right) r \mathrm{~d} r \\
& =\frac{1+\rho\left(\Gamma_{\mathrm{MR}}, \alpha\right)}{\lambda \pi R^{2} \mu\left(\Gamma_{\mathrm{RU}}, \alpha\right)}\left(1-\exp \left(-\frac{\lambda \pi R^{2} \mu\left(\Gamma_{\mathrm{RU}}, \alpha\right)}{1+\rho\left(\Gamma_{\mathrm{MR}}, \alpha\right)}\right)\right)
\end{aligned}
$$

Using Theorems 2 and 3, the following corollaries can be acquired.

Corollary 5. With $\sigma^{2}>0$, the coverage probability $p_{c_{-} \mathrm{RU}}$ increases with decreasing $P_{\mathrm{MR}}$ or increasing $P_{\mathrm{RU}}$.

Corollary 6. With $\sigma^{2}>0$, the coverage probability $p_{c_{-} \mathrm{RU}}$ increases with increasing $\Gamma_{\mathrm{MR}}$ or decreasing $\Gamma_{\mathrm{RU}}$. 
Corollary 7. When $\lambda_{R}<\lambda_{M}$, the coverage probability $p_{c \_ \text {RU }}$ decreases with increasing $\lambda_{R}$. When $\lambda_{R} \geq \lambda_{M}$, the coverage probability $p_{c \_\mathrm{RU}}$ remains the same with increasing $\lambda_{R}$.

Proof. When $\lambda_{R}<\lambda_{M}, \lambda=\lambda_{R}$, increasing $\lambda_{R}$ can increase $\lambda$ and decrease $p_{c_{-} \text {RU }}$. However, when $\lambda_{R} \geq \lambda_{M}$, $\lambda=\lambda_{M}$ as $\lambda_{R}$ rises, as a result, $p_{c_{-} \mathrm{RU}}$ remains the same with increasing $\lambda_{R}$.

\subsection{Power consumption model}

In this section, we model the power consumption of MBSs and RSs in downlink relay-assisted cellular networks. The relation between total power consumption $P_{\text {tot }}$ and transmit radiated power $P_{T}$ is modeled as [14-16] $P_{\text {tot }}=\beta P_{T}+$ $P_{0}$, where $1 / \beta$ is the efficiency of the power amplifier, and $P_{0}$ is the static power consumption, which includes signal processing overhead, battery backup, cooling power consumption, etc. As a result, for MBS and RS, the total power consumption $P_{M_{-} \text {tot }}, P_{R_{-} \text {tot }}$ can be given by

$$
\begin{aligned}
& P_{M_{-} \mathrm{tot}}=\beta_{M} P_{M}+P_{M 0} \\
& P_{R_{-} \mathrm{tot}}=\beta_{R} P_{R}+P_{R 0}
\end{aligned}
$$

where $1 / \beta_{M}, 1 / \beta_{R}$ denote the efficiency of the power amplifier for MBS and RS, $P_{M}$ and $P_{R}$ account for the total transmit power for MBS and RS, and $P_{M 0}$ and $P_{R 0}$ are the static power consumption for MBS and RS, respectively.

\subsection{Performance metrics}

We define the energy efficiency for the relay-assisted cellular networks as

$$
\begin{aligned}
\eta_{\mathrm{EE}} & =\frac{\text { Area spectral efficiency }}{\text { Average network area power consumption }} \\
& =\frac{\tau_{M}+\tau_{R}}{P_{M_{-} \text {ave }}+P_{R_{\_} \text {ave }}}(\text { bps } / \mathrm{Hz} / \mathrm{W})
\end{aligned}
$$

where $\tau_{M}$ and $\tau_{R}$ are the area spectrum efficiency over all the MBS-UE links and MBS-RS-UE links, respectively. $P_{M \_ \text {ave }}$ and $P_{R_{-} \text {ave }}$ denote the average network area power consumption for MBSs and RSs, respectively.

The throughput attained at the MBS-UE link is given by $p_{c_{-} \mathrm{MU}} \frac{w_{M}}{w_{M}+w_{R}} \log _{2}\left(1+\Gamma_{\mathrm{MU}}\right)$ where $\frac{w_{M}}{w_{M}+w_{R}}$ is the rate of occupancy of the frequency bands for the MBS-UE link. Thus, the area spectrum efficiency over all MBS-UE links is

$$
\tau_{M}=\lambda_{M} p_{c_{-} \mathrm{MU}} \frac{w_{M}}{w_{M}+w_{R}} \log _{2}\left(1+\Gamma_{\mathrm{MU}}\right) .
$$

The MBS sends messages to MBS-UE with transmit power $P_{\mathrm{MU}}$, and to the RS with transmit power $P_{\mathrm{MR}}$ only in the one of the two phrases, so the average macrocell network area power consumption is

$$
P_{M \_ \text {ave }}=\beta_{M}\left(\lambda_{M} P_{\mathrm{MU}}+\lambda P_{\mathrm{MR}} / 2\right)+\lambda_{M} P_{\mathrm{M} 0} .
$$

As at most $\lambda=\min \left(\lambda_{M}, \lambda_{R}\right)$ MBSs per square meter transmit signals to the RSs, the density used for $P_{\mathrm{MR}}$ is $\lambda$.

As a DF strategy is adopted by the RS, then the MBSRS-UE link operates only if both the relay and the user decode the messages received successfully, namely, the SINR attained at the RS and the R-UE must be larger than $\Gamma_{\mathrm{MR}}$ and $\Gamma_{\mathrm{RU}}$, respectively. Thus, the area spectrum efficiency for all the MBS-RS-UE links is given by

$$
\begin{aligned}
\tau_{R}= & \frac{1}{2} \lambda p_{c_{-} \mathrm{MR}} \times p_{c_{-} \mathrm{RU}} \frac{w_{R}}{w_{R}+w_{H}} \min \left\{\log _{2}\left(1+\Gamma_{\mathrm{MR}}\right),\right. \\
& \left.\log _{2}\left(1+\Gamma_{\mathrm{RU}}\right)\right\} .
\end{aligned}
$$

Note that the $1 / 2$ factor is due to half-duplex operation of the relay nodes, and $\frac{w_{R}}{w_{M}+w_{R}}$ is the rate of occupancy of the frequency bands for the MBS-RS-UE link.

At any time, the number of RSs taking part in the communication is at most the same as the number of MBSs ( $\lambda$ relays per square meters), and only the relays decoding signals for MBSs successfully forward signals to corresponding R-UEs $\left(\lambda p_{c}\right.$ MR relays per square meter), the other relays $\left(\lambda_{R}-\lambda p_{c_{-} \text {MR }}\right.$ relays per square meter) do not send signals but only consume the static power part. Thus, the average network area power consumption for RSs is as follows:

$$
P_{R_{\_} \mathrm{ave}}=\lambda p_{c_{\_} \mathrm{MR}}\left(\beta_{R} P_{R} / 2+P_{R 0}\right)+\left(\lambda_{R}-\lambda p_{c_{\_} \mathrm{MR}}\right) P_{\mathrm{R} 0} .
$$

Combining Equations 17 to 21, the expression of energy efficiency for the relay-assisted cellular networks is obtained.

The following theorem demonstrates the quasiconcavity of energy efficiency function.

Theorem 4. The energy efficiency function $\eta_{\mathrm{EE}}$ is strictly quasi-concave on $P_{\mathrm{MU}}$ or $P_{\mathrm{RU}}$.

Proof. We prove $\eta_{\mathrm{EE}}$ is strictly quasi-concave on $P_{\mathrm{MU}}$ here, and the process of the proof that $\eta_{\mathrm{EE}}$ is strictly quasi-concave on $P_{\mathrm{RU}}$ is similar. As defined in [17], a function $f: \mathbf{R}^{n} \rightarrow \mathbf{R}$ is said to be strictly quasi-concave if its sublevel set $S_{\alpha}=\{\mathbf{x} \mid \mathbf{x} \in \operatorname{dom} f, f(\mathbf{x}) \geq \alpha\}$ is strictly convex for every $\alpha$. When $\alpha \leq 0, S_{\alpha}$ is obviously convex on $P_{\text {MU }}$. When $\alpha>0, S_{\alpha}$ is equivalent to $S_{\alpha}=$ $\left\{P_{\mathrm{MU}} \mid \alpha\left(P_{M_{\_} \text {ave }}+P_{R_{-} \text {ave }}\right)-\tau_{M}-\tau_{R} \leq 0\right\}$. It can be easily proved that $p_{c_{-} \mathrm{MU}}$ is strictly concave on $P_{\mathrm{MU}}$, so $-\tau_{M}$ is strictly concave on $P_{\mathrm{MU}}$. Besides, $P_{M_{-} \text {ave }}$ linearly increases with $P_{\mathrm{MU}}, P_{R_{-} \text {ave }}$ and $\tau_{R}$ are not relevant with $P_{\mathrm{MU}}$. As a result, $S_{\alpha}$ is also strictly convex on $P_{\mathrm{MU}}$ and Theorem 4 follows. 
Due to the strict quasi-concavity of $\eta_{\mathrm{EE}}$ on $P_{\mathrm{MU}}$ or $P_{\mathrm{RU}}$, the optimal energy efficiency exists for various values of $P_{\mathrm{MU}}$ or $P_{\mathrm{RU}}$.

We now study the following optimization problem:

$$
\begin{gathered}
\min _{P_{\mathrm{MU}}} \eta_{\mathrm{EE}} \\
\text { s.t. } p_{c_{-} \mathrm{MU}} \geq \theta
\end{gathered}
$$

The above problem aims at finding the optimal energy efficiency under the constraint that the coverage probability for the MBS-UE link is larger than or equal to a certain threshold $\theta$. As $\eta_{\mathrm{EE}}$ is strictly quasi-concave on $P_{\mathrm{MU}}$, the bisection method can be used to find the optimal $\eta_{\mathrm{EE}}$ and the corresponding $P_{\mathrm{MU}}$ (denoted $\left.P_{\mathrm{MU} \_ \text {opt }}\right)$. If $p_{c_{-} \mathrm{MU}}\left(P_{\mathrm{MU} \_ \text {opt }}\right) \geq \theta$, then $\eta_{\mathrm{EE}}\left(P_{\mathrm{MU} \_o p t}\right)$ is the optimal value. Else, as $p_{c_{-} \mathrm{MU}}$ strictly increases monotonically on $P_{\mathrm{MU}}, P_{\mathrm{MU} \_o p t}$ is less than the power $\bar{P}_{\mathrm{MU} \_o p t}$ which makes $p_{c \_\mathrm{MU}}\left(\bar{P}_{\mathrm{MU} \_o p t}\right)=\theta$ and $\eta_{\mathrm{EE}}\left(\bar{P}_{\mathrm{MU} \_ \text {opt }}\right)$ is the optimal value according to the quasi-concavity of $\eta_{\mathrm{EE}}$. The detailed algorithm to find the optimal $\eta_{\mathrm{EE}}$ based on bisection method is described in Algorithm 1.

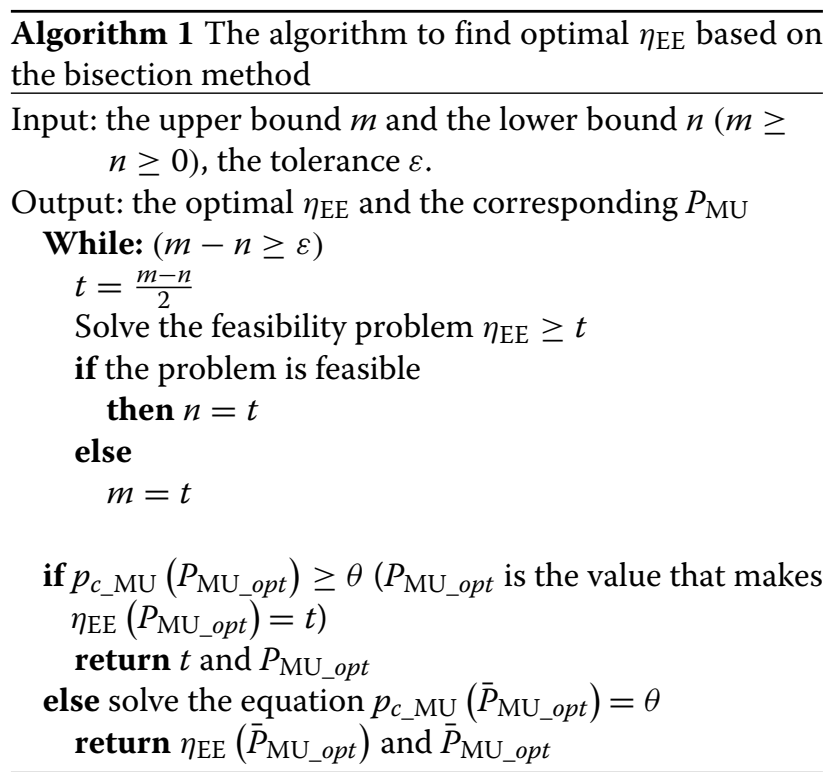

\section{Simulation results}

In this section, we evaluate the derived analytical results through simulation. We use the default values of the system model in Table 1 unless otherwise stated. The simulations are divided into two parts: coverage probability and energy efficiency.

\subsection{Simulation results for coverage probability}

In this part, we evaluate the relationship between coverage probability and transmit power of MBS or MBS density.
Table 1 Parameter values used in the simulations

\begin{tabular}{cc}
\hline Parameter & Value \\
\hline$\lambda_{M}, \lambda_{R}$ & $10^{-5} \mathrm{~m}^{-2}, 10^{-4} \mathrm{~m}^{-2}$, \\
$P_{\mathrm{MU}}, P_{\mathrm{RU}}, P_{\mathrm{MR}}$ & $43 \mathrm{dBm}, 30 \mathrm{dBm}, 33 \mathrm{dBm}$ \\
$R$ & $40 \mathrm{~m}$ \\
$\alpha$ & 4 \\
$W_{M}, W_{R}$ & $40 \mathrm{MHz}, 4 \mathrm{MHz}$ \\
$\Gamma_{\mathrm{MR}}, \Gamma_{\mathrm{RU}}, \Gamma_{\mathrm{MR}}$ & $-10 \mathrm{~dB},-10 \mathrm{~dB},-10 \mathrm{~dB}$ \\
$\sigma^{2}$ & $-60 \mathrm{dBm}$ \\
$\beta_{M}, \beta_{R}$ & $5.32,4.8$ \\
$P_{\mathrm{M} 0}, P_{\mathrm{R} 0}$ & $118.7 \mathrm{~W}, 7.5 \mathrm{~W}$ \\
\hline
\end{tabular}

Figure 3 shows the coverage probability for the MBS-UE link $p_{c \_}$MU versus the transmit power of MBS $P_{\mathrm{MU}}$ for different target SINRs $\Gamma_{\mathrm{MU}}$. It can be seen from the figure that the coverage probability increases with the transmit power, and the growth rate is high when the transmit power is relatively small, whereas it approaches zero when the transmit power is relative large. Besides, as the target SINR increases, the coverage probability decreases, and higher target SINR results in larger changes of coverage probability with the variation of transmit power. Note that for $\Gamma_{\mathrm{MU}}=-20 \mathrm{~dB}$, the curve is almost flat, while for $\Gamma_{\mathrm{MU}}$ up to $0 \mathrm{~dB}$, the curve becomes the most curved.

Figure 4 plots the coverage probability for the MBS-RS link $p_{c}$ MR versus the MBS density $\lambda_{M}$ for different values of pathloss exponent $\alpha$. The curves can be divided into two parts due to the value of $\lambda$. When $\lambda_{M}$ is smaller than that of RS $\lambda_{R}$, $\lambda$ equals to $\lambda_{M}$, and for pathloss exponent increasing, the curves become more and more curved. When the pathloss exponent is 3 , the coverage probability remains almost the same as the density changes in this range. However, when the pathloss exponent becomes 4 or 5 , the coverage probability increases as the density grows. Moreover, when $\lambda_{M} \geq \lambda_{R}, \lambda$ equals to the constant value $\lambda_{R}$, then all the curves increase slowly with $\lambda_{M}$.

The relationship between coverage probability for RSUE link $p_{c \_ \text {RU }}$ and density of MBS $\lambda_{M}$ for different pathloss exponents $\alpha$ is shown in Figure 5. Compared to $p_{c_{-} \text {MR }}$ or $p_{c_{-} \mathrm{RU}}$, the expression of $p_{c_{-} \mathrm{RU}}$, which is relevant to $p_{c_{\_} \mathrm{MR}}$ and the density of both MBSs and RSs, is quite different and causes different curve shapes. When $\lambda_{M}$ is smaller than $\lambda_{R}$, the value of $\lambda$ is $\lambda_{M}$, and the coverage probabilities almost linearly decrease as $\lambda_{M}$ increases. However, when $\lambda_{M}$ becomes larger than $\lambda_{R}$, the value of $\lambda$ is the constant value $\lambda_{R}$, and then the coverage probabilities just slightly decrease as the density of MBS increases. This is because when $\lambda_{M}$ becomes larger than $\lambda_{R}, p_{c_{-}} \mathrm{RU}$ only varies with $p_{c_{-}} \mathrm{MR}$. From Figure 4 , we can see that $p_{c_{-} \mathrm{MR}}$ 


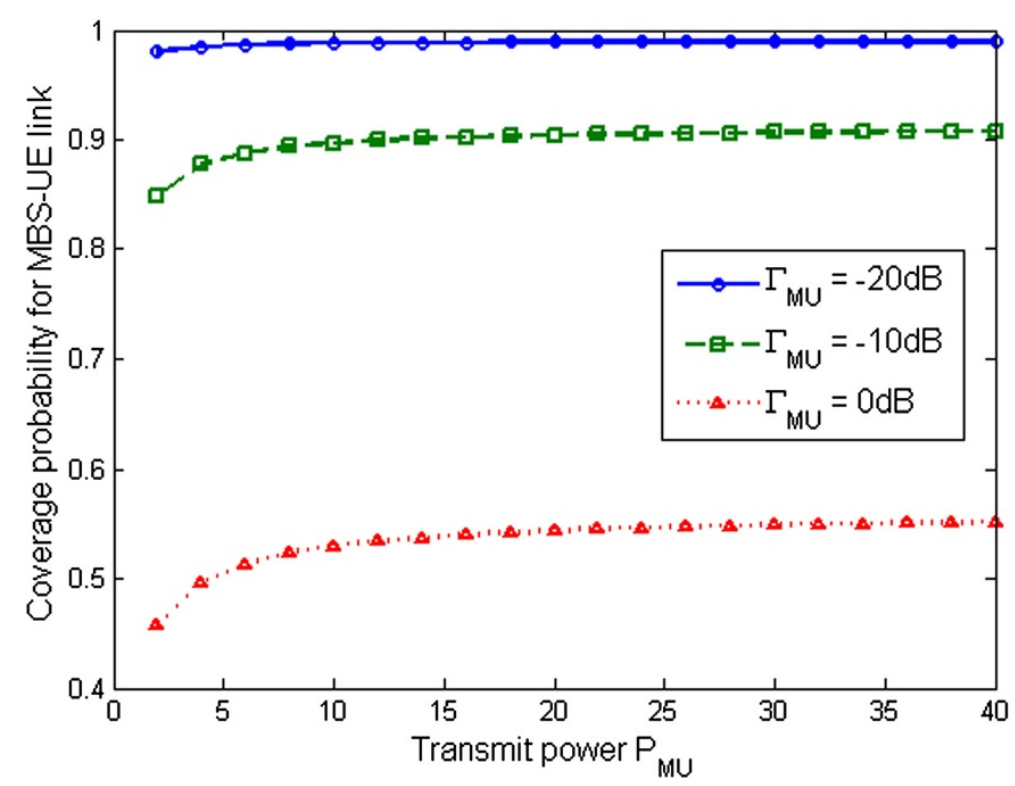

Figure 3 Coverage probability for the MBS-UE link vs. transmit power of MBS for different target SINR.

slightly increases when $\lambda_{M}$ is larger than $\lambda_{R}$, which results in the shape of $p_{c_{-}} \mathrm{RU}$.

\subsection{Simulation results for energy efficiency}

The energy efficiency for downlink relay-assisted cellular networks is simulated in this part. Figure 6 shows how the energy efficiency varies with the density of MBSs for different densities of RSs. It can be seen that the energy efficiency increases with the density of MBSs and saturates when the density goes to infinity. That is to say, the growth of area spectral efficiency is faster than the growth of average network power consumption as the density of MBSs increases. But when the density of MBSs is relatively high, continuously increasing MBSs cannot further increase the energy efficiency. In addition, higher density of RSs brings about lower energy efficiency in the case of

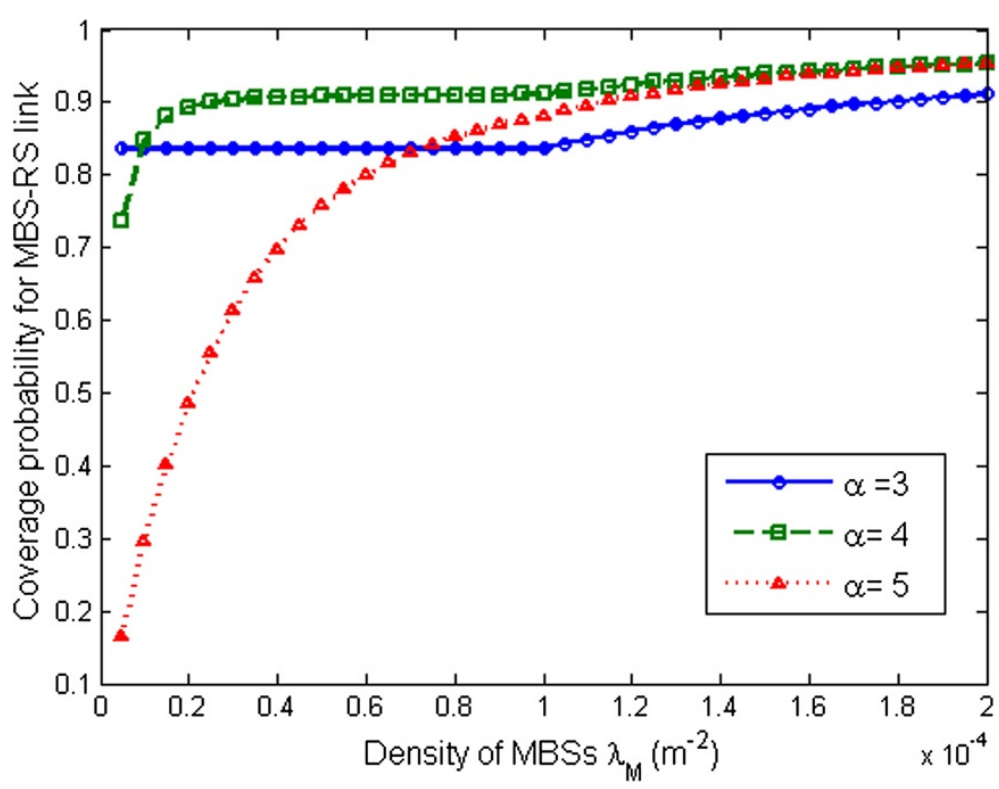

Figure 4 Coverage probability for the MBS-RS link vs. density of MBSs for different pathloss exponents. 


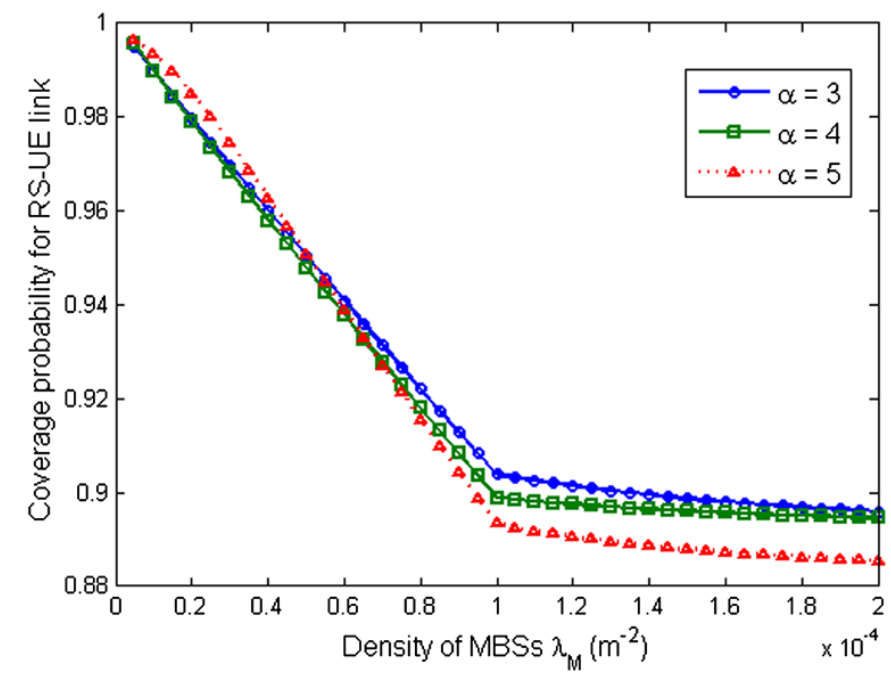

Figure 5 Coverage probability for the RS-UE link vs. density of MBSs for different pathloss exponents.

all other parameters constant. This is due to the access assumption in our model. We assume that each MBS serves at most one M-UE and RS at any time, and also, each RS serves one R-UE at any time. As a result, if the density of RSs is larger than that of MBSs, on average only $\lambda_{M}$ RSs per square meter can be served at a time, the area spectral efficiency remains the same but the average area power consumption, mainly the average area static power consumption of RSs increases with the growth of density of RSs.
Figure 7 displays the energy efficiency with respect to transmit power of MBS $P_{\mathrm{MU}}$ for different values of RS densities. As predicted in Theorem 4, the energy efficiency first increases and then decreases, showing a quasi-convex trend as the transmit power varies. The optimal energy efficiency can be found using the algorithm shown in Table 1. If the threshold $\theta$ is set to zero and the tolerance $\varepsilon$ is $10^{-5}$, the optimal energy efficiency is $6.40 \times 10^{-4} \mathrm{bps} / \mathrm{Hz} / \mathrm{W}, 5.26 \times 10^{-4} \mathrm{bps} / \mathrm{Hz} / \mathrm{W}$ and $4.48 \times 10^{-4} \mathrm{bps} / \mathrm{Hz} / \mathrm{W}$ corresponding to the three

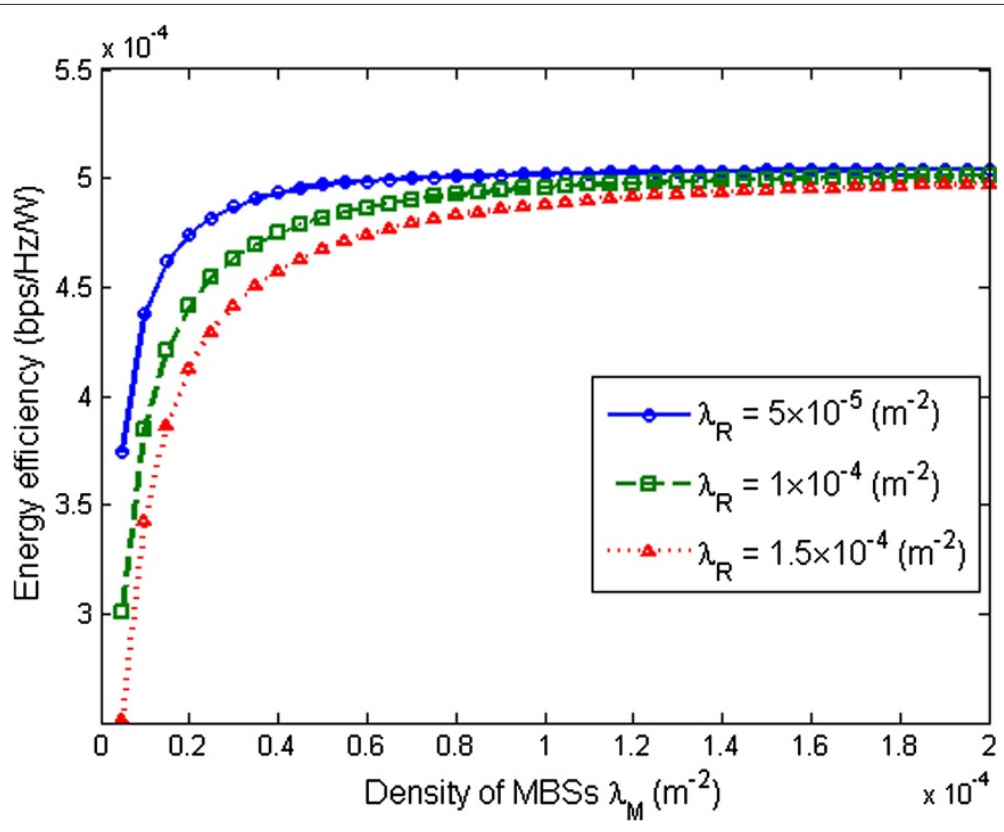

Figure 6 Energy efficiency vs. density of MBSs for different density of RSs. 


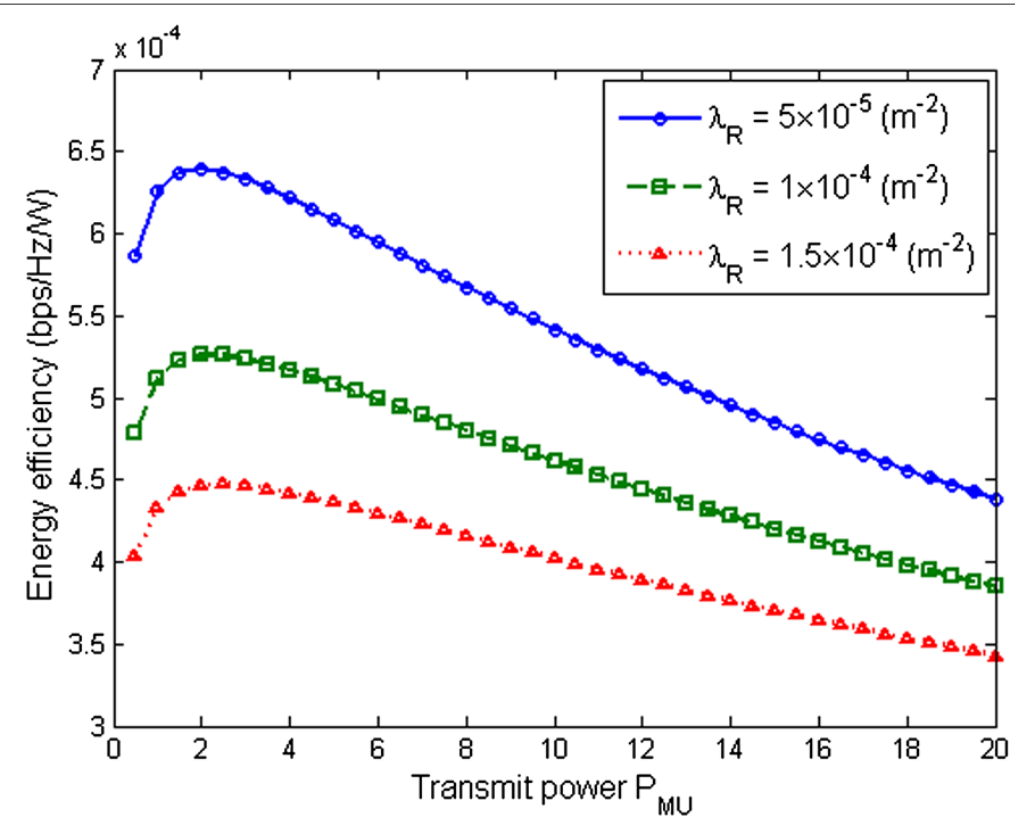

Figure 7 Energy efficiency vs. transmit power of MBS for different density of RSs.

curves when $\lambda_{R}=5 \times 10^{-5} \mathrm{~m}^{-2}, 1 \times 10^{-4} \mathrm{~m}^{-2}, 1.5 \times$ $10^{-4} \mathrm{~m}^{-2}$, respectively. Besides, the figure also shows that while the density of RSs grows, the energy efficiency drops, which means that the growth of area spectrum efficiency by increasing the density of RSs cannot compensate for the growth of average network power consumption.

\section{Conclusions}

In this paper, we study the energy efficiency of downlink relay-assisted cellular networks where MBSs and RSs are distributed according to independent HPPPs. First, we build on a tractable stochastic geometry-based model to derive the coverage probabilities for MBSUE, MBS-RS, and RS-UE links. Second, using power consumption models for both macrocell and relay networks, we obtain expressions for the network energy efficiency. Our results reveal that there is a fundamental tradeoff between energy efficiency and transmit MBS power, and the main takeaway is that deploying more MBSs generally triggers higher energy efficiency; however this gain hits a ceiling as the density of MBSs increases.

\section{Competing interests}

The authors declare that they have no competing interests.

\section{Acknowledgements}

This work is supported by the National Basic Research Program of China (No. 2012CB316000), the National Natural Science Foundation of China (No. 61201192), the National S\&T Major Project (No. 2013ZX03004007), International Science and Technology Cooperation Program (No.
2012DFG12010), China's 863 Project (No. 2012AA01 1402), Keygrant Project of Chinese Ministry of Education (No. 313005), the Open Research Fund of National Mobile Communications Research Laboratory, Southeast University (No. 2012D02), and Tsinghua-Qualcomm Joint Research Program.

\section{Author details}

${ }^{1}$ Department of Electronic Engineering, Tsinghua University, Beijing 100084, China. ${ }^{2}$ Research Institute of Information Technology, State Key Laboratory on Microwave and Digital Communications, Tsinghua National Laboratory for Information Science and Technology(TNList), Tsinghua University, 100084 Beijing, China. ${ }^{3}$ SUPELEC, Department of Telecommunications, 3 rue Joliot-Curie, Gif-sur-Yvette 91192, France.

Received: 21 September 2013 Accepted: 7 March 2014

Published: 14 March 2014

\section{References}

1. JN Laneman, DNC Tse, GW Wornell, Cooperative diversity in wireless networks: efficient protocols and outage behavior. IEEE Trans. Inf. Theory. 50(12), 3062-3080 (2004)

2. GY Li, Z Xu, C Xiong, C Yang, S Zhang, Y Chen, S Xu, Energy-efficient wireless communications: tutorial, survey, and open issues. IEEE Wireless Commun. Mag. 18(6), 28-35 (2011)

3. $\mathrm{H} Y \mathrm{Yu}, \mathrm{H}$ Qin, $Y \mathrm{Li}, \mathrm{Y} Z \mathrm{Zhao}, \mathrm{XXu}$, J Wang, Energy-efficient power allocation for non-regenerative OFDM relay links. Sci. China Information Sci. 56(2), 185-192 (2013)

4. C Sun, C Yang, Is two-way relay more energy efficient? in Proceedings of the Global Telecommunications Conference (GLOBECOM 2011), 2011 IEEE (IEEE Houston, Texas, USA, 2011), pp. 1-6

5. C Sun, C Yang, Energy efficient hybrid one-way and two-way relay transmission strategy, in Proceedings of the Acoustics, Speech and Signal Processing (ICASSP), 2012 IEEE International Conference on (IEEE Kyoto, Japan, 2012), pp. 2497-2500

6. H Yu, Y Li, X Zhong, L Wang, J Wang, The analysis of the energy efficiency for the decode-and-forward two-way relay networks, vol. 2013 (IEEE, Shanghai, China, 2013), pp. 2823-2827

7. R Madan, N Mehta, A Molisch, J Zhang, Energy-efficient cooperative relaying over fading channels with simple relay selection. IEEE Trans. Wireless Commun. 7(8), 3013-3025 (2008) 
8. R Huang, C Feng, T Zhang, W Wang, Energy-efficient relay selection and power allocation scheme in AF relay networks with bidirectional asymmetric traffic, in Proceedings of the Wireless Personal Multimedia Communications (WPMC), 2011 14th International Symposium on (IEEE Brest, France, 2011), pp. 1-5

9. JG Andrews, F Baccelli, RK Ganti, A tractable approach to coverage and rate in cellular networks. IEEE Trans. Commun. 59(11), 3122-3134 (2011)

10. YS Soh, TQS Quek, M Kountouris, H Shin, Energy efficient heterogeneous cellular networks. IEEE J. Selec. Areas Commun. 31(5), 840-850 (2013)

11. N Deng, S Zhang, W Zhou, J Zhu, A stochastic geometry approach to energy efficiency in relay-assisted cellular networks, in Proceedings of the Global Telecommunications Conference (GLOBECOM 2012), 2012 IEEE (IEEE Anaheim, California, USA, 2012), pp. 3484-3489

12. H Wei, N Deng, M Zhao, W Zhou, P Dong, Station density effect on energy efficiency of relay-assisted cellular networks, in Proceedings of the 1st IEEE International Conference on Communications in China (ICCC) (IEEE Beijing, China, 2012), pp. 411-415

13. D Stoyan, WS Kendall, J Mecke, L Ruschendorf, Stochastic Geometry and Its Applications, Volume 2, Chichester, UK. (Wiley, Chichester, 1995)

14. G Auer, V Giannini, C Desset, I Godor, P Skillermark, M Olsson, MA Imran, D Sabella, MJ Gonzalez, O Blume, A Fehske, How much energy is needed to run a wireless network? IEEE Wireless Commun. Mag. 18(5), 40-49 (2011)

15. AJ Fehske, F Richter, GP Fettweis, Energy efficiency improvements through micro sites in cellular mobile radio networks, in GLOBECOM Workshops, 2009 IEEE (IEEE, Hawaii, USA, 2009), pp. 1-5

16. O Arnold, F Richter, G Fettweis, O Blume, Power consumption modeling of different base station types in heterogeneous cellular networks, in Future Network and Mobile Summit, 2010 (IEEE Florence, Italy, 2010), pp. 1-8

17. SP Boyd, L Vandenberghe, Convex Optimization. (Cambridge University Press, Cambridge, UK, 2004)

doi:10.1186/1687-6180-2014-32

Cite this article as: Yu et al.: Energy efficiency analysis of relay-assisted cellular networks. EURASIP Journal on Advances in Signal Processing 2014 2014:32.

\section{Submit your manuscript to a SpringerOpen ${ }^{\circ}$ journal and benefit from:}

- Convenient online submission

- Rigorous peer review

- Immediate publication on acceptance

- Open access: articles freely available online

- High visibility within the field

- Retaining the copyright to your article

Submit your next manuscript at $\boldsymbol{\nabla}$ springeropen.com 